\title{
Supporting Information: Exciton Spatial Dynamics and Self-Trapping in Carbon Nanocages
}

\author{
Beatriz Rodríguez-Hernández, ${ }^{\dagger}$ Tammie Nelson, ${ }^{\ddagger}$ Nicolas Oldani ${ }^{\dagger}$ Aliezer \\ Martínez-Mesa, ${ }^{\dagger}$, Llinersy Uranga-Piña, ${ }^{\dagger} \uparrow$ Yasutomo Segawa, ${ }^{\S, \|, \perp, \# ~ S e r g e i ~}$ \\ Tretiak,,$^{\ddagger} @$ Kenichiro Itami, ${ }^{\S}, \|, \triangle$ and Sebastian Fernandez-Alberti, ${ }^{*} \dagger$
}

†Departamento de Ciencia y Tecnologia, Universidad Nacional de Quilmes/CONICET, B1876BXD Bernal, Argentina

$\ddagger$ Physics and Chemistry of Materials, Theoretical Division, Los Alamos National Laboratory, Los Alamos, NM, 87545, United States

\DynAMoS (Dynamical processes in Atomic and Molecular Systems), Facultad de Fsica, Universidad de La Habana, San Lzaro y L, La Habana 10400, Cuba.

§Graduate School of Science, Nagoya University, Nagoya 464-8602, Japan

\|JST, ERATO, Itami Molecular Nanocarbon Project, Nagoya University, Nagoya

$$
\text { 464-8602, Japan }
$$

$\perp$ Institute for Molecular Science, Myodaiji, Okazaki, 444-8787, Japan

\#Department of Structural Molecular Science, SOKENDAI (The Graduate University for Advanced Studies), Myodaiji, Okazaki, 444-8787, Japan

@ Center for Integrated Nanotechnologies, Los Alamos National Laboratory, Los Alamos, NM, 87545, United States

$\triangle$ Institute of Transformative Bio-Molecules (WPI-ITbM) Nagoya University, Nagoya 464-8602, Japan

E-mail: sfalberti@gmail.com 


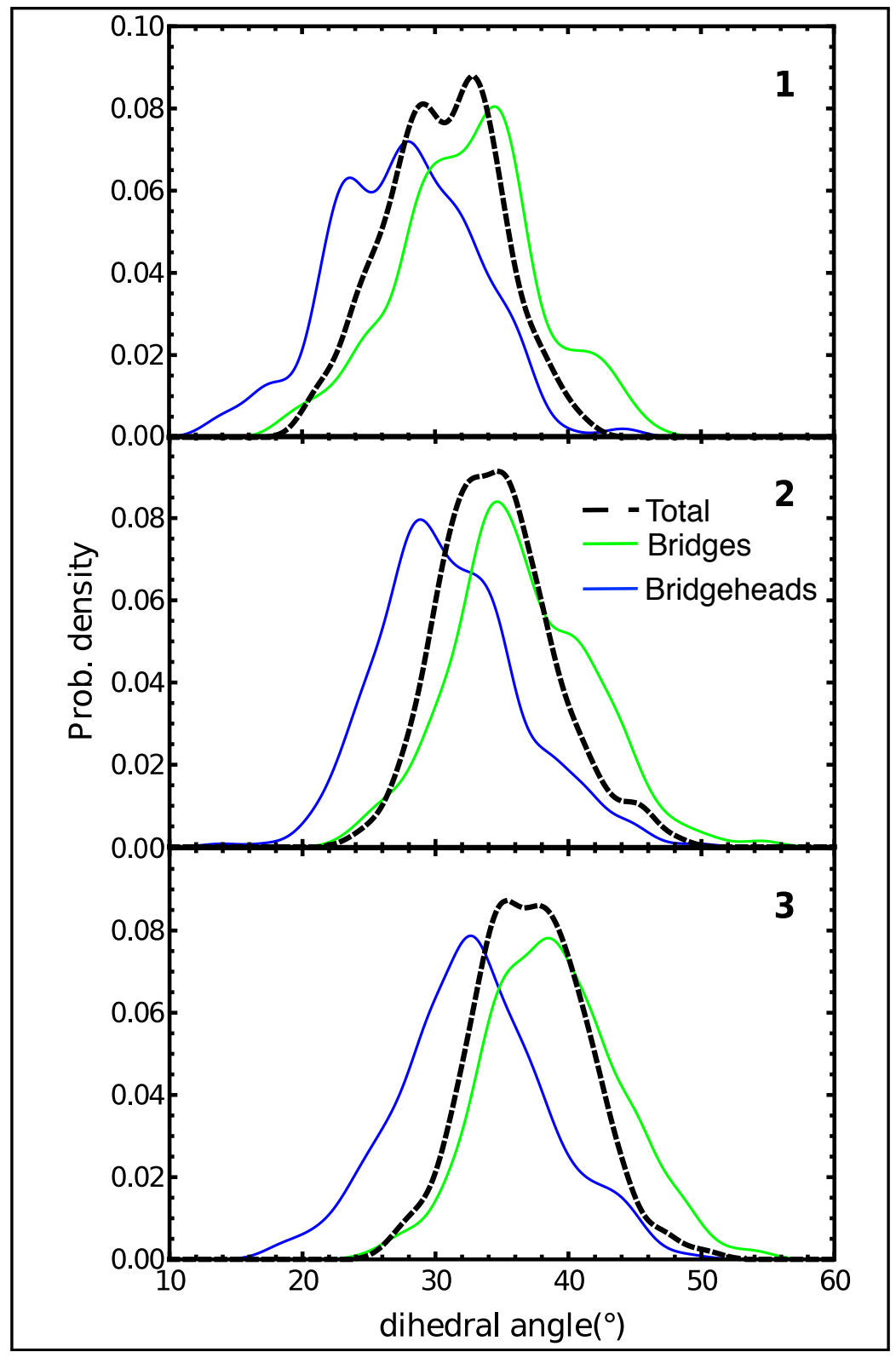

Figure S 1: Probability density distributions of average dihedral angles between neighboring phenyl rings obtained from equilibrated ground-state configurations at $300 \mathrm{~K}$ for $\mathbf{1}, \mathbf{2}$, and 3. The separate contributions to the total distribution (black dashes) are plotted for the dihedral angles between a bridgehead phenyl and a neighboring phenyl of the bridge (blue) and between two phenyl rings belonging to the bridges (green). Bridgehead phenyl rings are more constrained and therefore exhibit lower dihedral angles on average compared to bridge phenyl rings, giving rise to the double peak in the total distribution. 


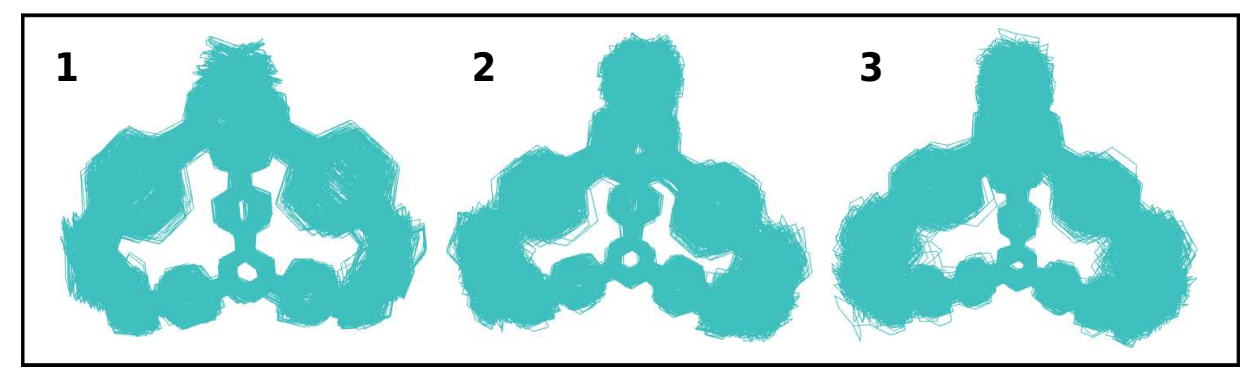

Figure S 2: superposition of molecular configurations collected from ground state conformational samplings at $300 \mathrm{~K}$ for $\mathbf{1 , 2}$, and $\mathbf{3}$. The conformational disorder increases with nanocage size. The broader conformational range in larger nanocages is a consequence of the increased backbone flexibility from the longer $[n]$ paraphenylene chains.
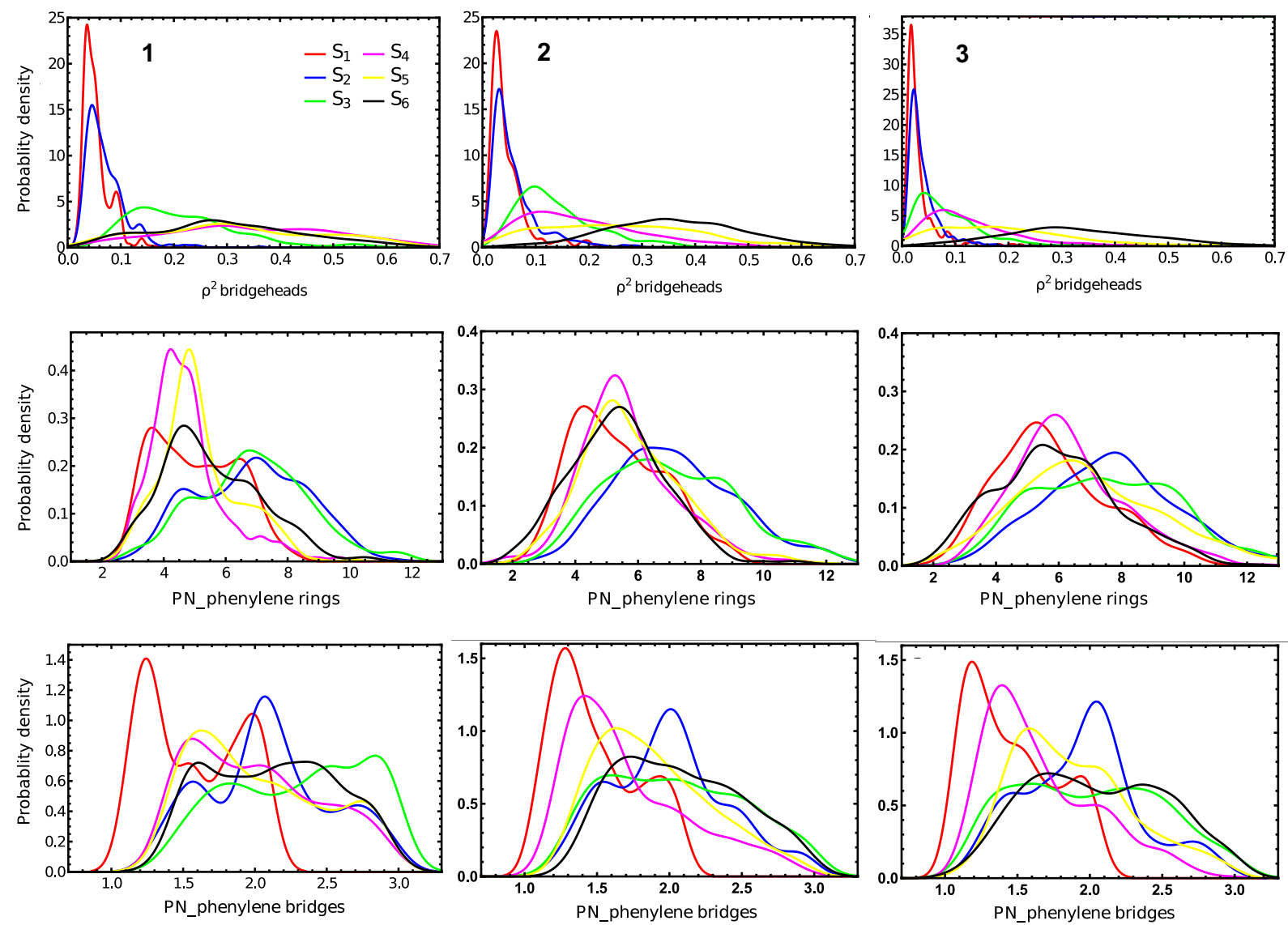

Figure S 3: Analysis of the spatial localization of the electronic transition density for the different excited states evaluated for the ensemble of the initial equilibrated ground state conformation (400 samples) for carbon nanocage $\mathbf{1}$ (left column), $\mathbf{2}$ (middle column), 3 (right column). (top row) distribution of the transition density fraction localized on the two bridgeheads; (middle row) distribution of participation number for phenylene rings $\left(P N_{\text {phenylenerings }}(t=0)\right)$; (bottom row) distribution of participation number for bridges $\left(P N_{\text {phenylenebridges }}(t=0)\right)$. 


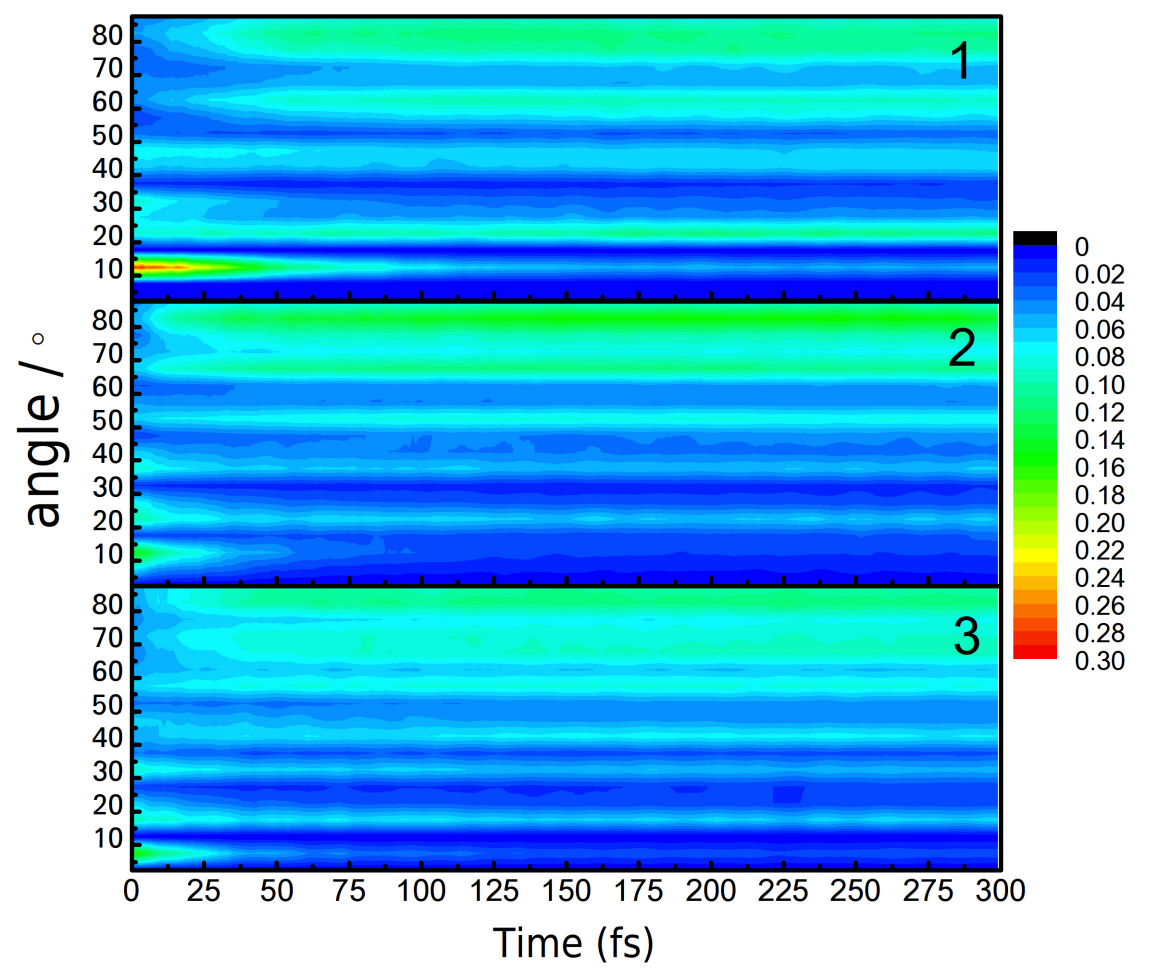

Figure S 4: The change in the angular distribution of the spatial localization of the transition density during NEXMD simulations of carbon nanocages $\mathbf{1}, \mathbf{2}$, and $\mathbf{3}$. The angles are calculated with respect to a vector in the direction of the axis that connects the two bridgeheads and centered in the center of mass of the nanocage. The color bar indicates the fraction of the transition density. The initial exciton localization at $\sim 10^{\circ}$ points to a localization near the bridgeheads. The exciton then experiences an ultrafast migration towards the center of the phenylene bridges, denoted by angles $\sim 90^{\circ}$. 


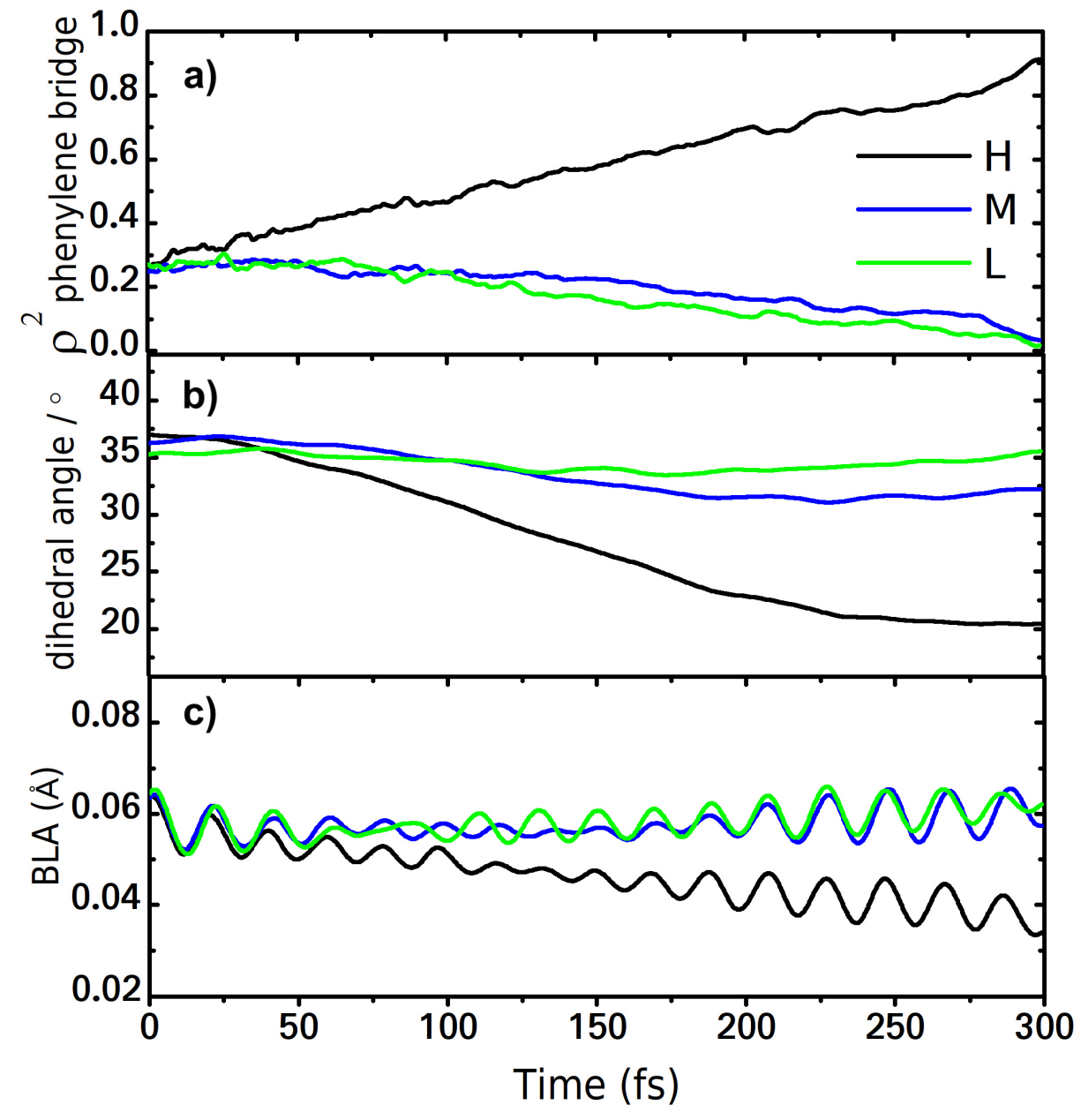

Figure S 5: Time evolution of different electronic and structural features for High (H), Middle $(\mathrm{M})$, and Low (L) phenylene bridges in carbon nanocage $\mathbf{2}$ during NEXMD simulations. $\mathrm{H}$, $\mathrm{M}$, and $\mathrm{L}$ correspond to phenylene bridges with decreasing final values of $\rho_{\text {phenylenebridge }}^{2}$. (a) fraction of transition density; (b) average dihedral angle between neighboring phenylene bridge units; (c) average bond length alternation values along the bridge. 


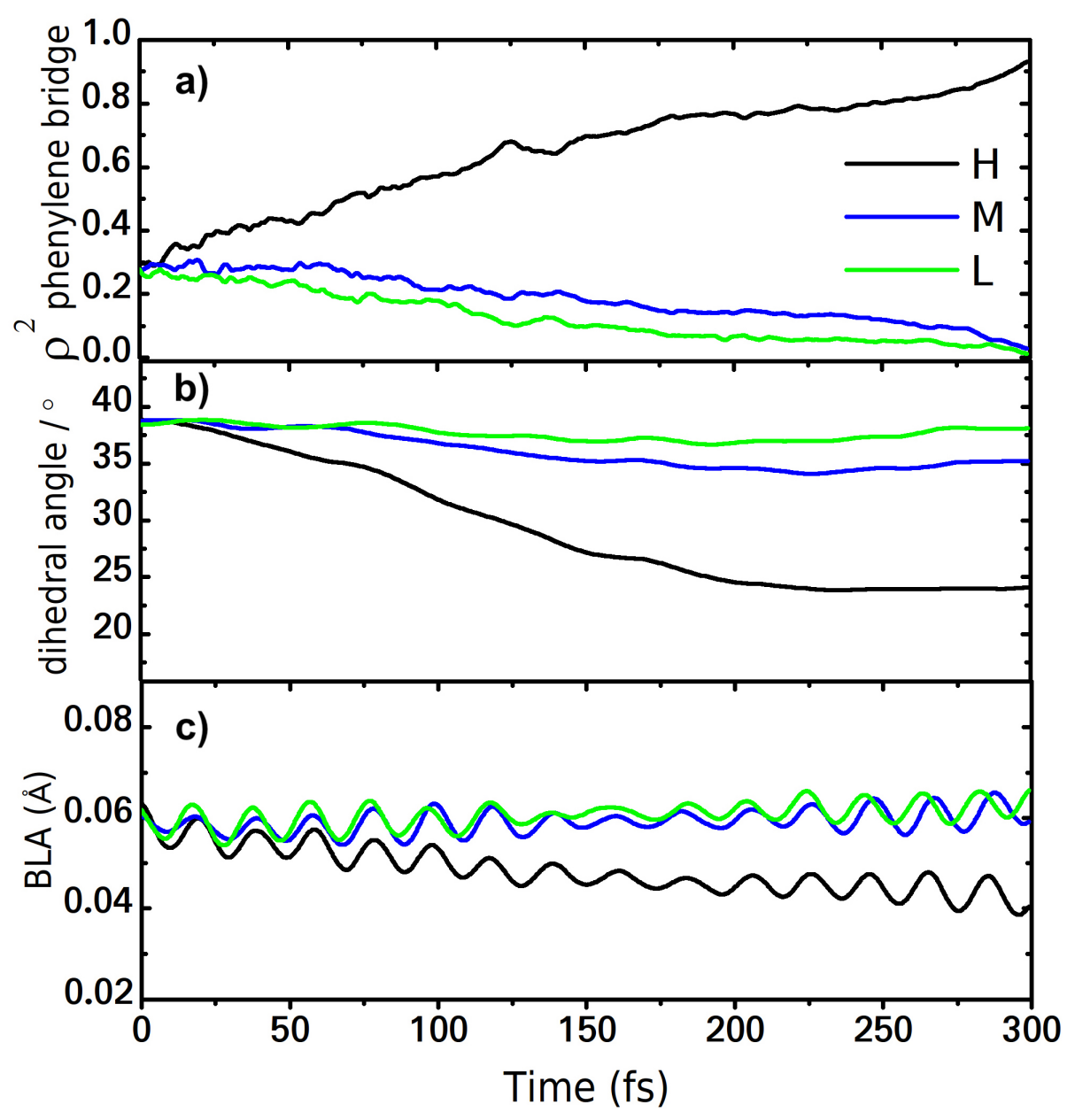

Figure S 6: Time evolution of different electronic and structural features for High (H), Middle $(\mathrm{M})$, and Low (L) phenylene bridges in carbon nanocage 3 during NEXMD simulations. H, $\mathrm{M}$, and $\mathrm{L}$ correspond to phenylene bridges with decreasing final values of $\rho_{\text {phenylenebridge }}^{2}$. (a) fraction of transition density; (b) average dihedral angle between neighboring phenylene bridge units; (c) average bond length alternation values along the bridge. 


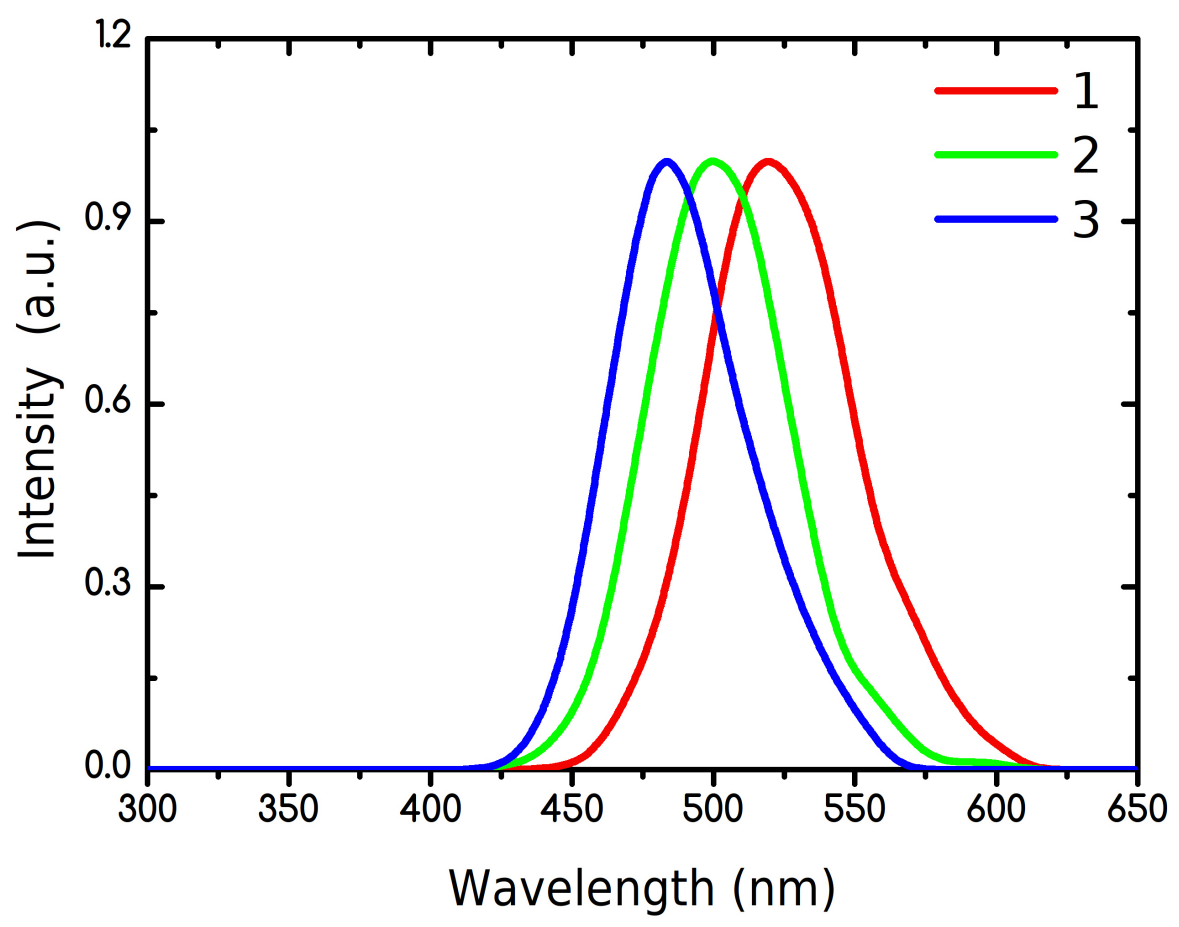

Figure S 7: Simulated emission spectra for $\mathbf{1}, \mathbf{2}$, and $\mathbf{3}$ calculated for the collected set of final structures and weighted according to the respective $\mathrm{S}_{1}$ oscillator strengths. 